\title{
Synthesis of Amine Terminated Pegylated Iron Oxide Nanoparticles for Prospective Astrocytoma Resection Grade Improvement
}

Isaias Eduardo Hernández-Verdin ${ }^{1}$, María Luisa Martínez², Alejandro Miranda-González ${ }^{3}$, Mónica Alicia Sierra del Río ${ }^{3}$, Oracio Serrano-Torres ${ }^{4}$ and Mario Ávila-Rodríguez ${ }^{4}$

1. Experimental neuro-oncology, Groupe Hospitalier Pitié-Salpêtrière, Paris 75013, France

2. Departamento de Ciencias e Ingeniería, Universidad Iberoamericana León, León 37238, Mexico

3. Departamento de Neurociencias, Hospital Regional de Alta Especialidad del Bajío, León 37660, Mexico

4. Departamento de Química, División de Ciencias Naturales y Exactas, Campus Guanajuato, Universidad de Guanajuato, Guanajuato 36040, Mexico

\begin{abstract}
Having a survival rate to 5 years of only 3\%, Glioblastoma's (GBM) main treatment is surgical excision. Iron oxide nanoparticles have been proved to be a magnetic resonance imaging contrast agent and, if synthesized and tuned correctly, could be used to improve complete GBM resection. In this work monodisperse iron oxide nanoparticles were synthesized using thermal decomposition method, then a ligand exchange reaction with 3-aminopropyl trimethoxysilane (APS) was performed, following Pegylation of the particles using dicarboxylic acid PEG (PEG-diacid) and finally aminating with 2,2'-(ethylenedioxy) bis(ethylamine), last two by amide reactions. STEM and DLS demonstrate monodispersity $(\log \sigma<0.2)$ and desired size range to penetrate the blood-brain barrier (BBB); FT-IR shows the reactions were executed correctly and finally stability in deionized water, $0.07 \mathrm{M} \mathrm{NaCl}$ and PBS $1 \mathrm{X}$, as a function of 0-30 days, was tested. Results revealed the importance that the oleic acid/iron oleate molar ratio and the growth stage time represents for determining iron oxide nanoparticles' size; as well as APS concentration and nucleation time influence on silica coating when performing the ligand exchange reaction. The produced iron oxide nanoparticles exhibit stability and proper amine terminated groups which are needed to allow easy incorporation of Chlorotoxin, a 36-amino acid peptide that binds specifically to astrocytoma cells, and a fluorescent molecule, which enables real time visualization of the tumor during surgery.
\end{abstract}

Key words: Iron oxide nanoparticles, silanization, pegylation, astrocytoma.

\section{Nomenclature}

GBM: glioblastoma

IO: $\quad$ iron oxide

NPs: nanoparticles

MRI: magnetic resonance image

BBB: blood-brain barrier

APS: $\quad 3$-aminopropyl trimethoxysilane

PEG-diacid: dicarboxylic acid poly (ethylene glycol)

EDEA: 2,2'-(ethylenedioxy) bis(ethylamine)

STEM: $\quad$ scanning transmission electron microscopy

DLS: dynamic light scattering

FT-IR: $\quad$ Fourier transform infrared spectroscopy

IO-OA: $\quad$ iron oxide-oleic acid

\section{Greek letters}

$\sigma: \quad$ standard deviation

\section{Introduction}

Astrocytomas are a type of brain tumor which can be presented in four different grades and are deadly in most of the cases. Even though, Glioblastoma (GBM), represents $79 \%$ of all astrocytoma's cases and 53.9\%

Corresponding author: Isaias Eduardo Hernández-Verdin, MSc, research fields: nanomaterials, neuro-oncology bioinformatics. 
of glioma's; surgical excision is still the only main treatment for this disease [1]. However, if an extensive resection is performed a biological function loss could happen; on the other hand, incomplete resection leaves more residual malignant cells, that is translated in more post posttreatment sessions. Both outcomes are undesirable, the first by marking the patient with a biological disability and the second by strongly impacting the family's economy [2].

Nanotechnology has gained attention thanks to its high efficiency and the possibility to make composite materials exhibiting multiple properties [3, 4]. Iron oxide (IO) nanoparticles (NPs) can: be used for preoperative diagnosis of astrocytomas using MRI (magnetic resonance image) [5], penetrate the blood-brain barrier (BBB) [6, 7], demonstrate physiological stability if pegylated [6, 8] and allow delimitation and real time visualization of the tumor with a fluorescent molecule attached [9]. Furthermore, astrocytoma cells affinity to up to $90 \%$ in GBM is guaranteed if Chlorotoxin, a 36 amino acid peptide that binds to the matrix metalloproteinase-2, is incorporated to the nanoparticle surface [7-9].

Different methods are available to synthesize MRI contrast agent IO NPs; however, it is known that the thermal decomposition method allows a more precise control on monodispersity and hence less variation on the nanoparticles physical-chemical properties [10-12]. Nonetheless, these NPs are hydrophobic and require a ligand exchange reaction to acquire hydrophilicity. IO nanoparticles' silanization followed by pegylation grants steric stabilization by competing against Van Der Waals and magnetic attraction forces [13, 14], biocompatibility and bloodstream half-life increment [15].

In this work we present the synthesis of monodisperse IO NPs using thermal decomposition method (with different oleic acid/iron oleate rates and reflux times), followed by ligand exchange reaction with 3-aminopropyl trimethoxysilane (APS) (with different reaction times and APS concentration), then pegylation of the particles using dicarboxylic acid Poly (ethylene glycol) (PEG-diacid) and finally amination with 2,2'-(ethylenedioxy) bis(ethylamine) (EDEA) to allow easy incorporation of chlorotoxin and a fluorescent molecule.

\section{Experimental Setup}

\subsection{Materials}

All products and solvents were used as received. Sodium hydroxide, oleic acid, sodium chloride, ferric chloride, hexane, 1-octadecene, acetone, isopropanol, 3-aminopropyl trimethoxysilane, acetic acid, poly (ethylene glycol) (4,000 molecular weight), chromium trioxide, sulfuric acid and 2,2'-(ethylenedioxy) bis(ethylamine) were purchased from Sigma Aldrich.

\subsection{Oxidation of PEG to PEG-Dicarboxylic Acid}

To oxidize the hydroxyl groups in PEG, 40 g was dissolved in $400 \mathrm{~mL}$ of acetone and then $17 \mathrm{~mL}$ of Jone's reagent was added (35 g of chromium trioxide in $250 \mathrm{~mL}$ of distilled water followed by slow addition of $30 \mathrm{~mL}$ of sulfuric acid). The reaction was left for 24 $\mathrm{h}$ under stirring at room temperature and then quenched by adding $5 \mathrm{~mL}$ of isopropanol. Chromium salts were removed with filtration under vacuum. The obtained clear acetone solution was distillated and desiccated to finally recover a green powder. Carboxylic acid conversion was estimated by titration of one gram of PEG-diacid dissolved in $10 \mathrm{~mL}$ of water with $0.0168 \mathrm{~N} \mathrm{NaOH}$ and two drops of phenolphthalein [16].

\subsection{Synthesis of Iron Oxide-Oleic Acid (IO-OA) NPs}

First, the precursors iron oleate and sodium oleate were synthesized. Sodium oleate was synthesized by mixing $5.084 \mathrm{~g}$ of oleic acid with solution 1:1 water:ethanol of $\mathrm{NaOH}(5 \mathrm{~g}$ in $30 \mathrm{~mL}$ ) and stirring at $70{ }^{\circ} \mathrm{C}$ for $40 \mathrm{~min}$. Then, $75 \mathrm{~mL}$ of $1 \mathrm{M} \mathrm{NaCl}$ was added and stirred until a yellow paste was formed followed by filtration under vacuum. Secondly, iron oleate was obtained by mixing $4 \mathrm{mmol}$ of ferric 
chloride, $12 \mathrm{mmol}$ of sodium oleate, $5 \mathrm{~mL}$ of distilled water and $14 \mathrm{~mL}$ of hexane and stirring to reflux at $60{ }^{\circ} \mathrm{C}$ for $4 \mathrm{~h}$. Subsequently, the product was washed several times with $12 \mathrm{~mL}$ of water in a decantation funnel and thoroughly desiccated to finally obtain a reddish paste.

IO-OA NPs were prepared by thermal decomposition of iron oleate $(0.21 \mathrm{mmol})$ in 1-octadecene $(6.5 \mathrm{~mL})$ in the presence of " $\mathrm{n}$ " mmol of oleic acid at different oleic acid/iron oleate rates: 1.5 (M1), 3 (M2), 4.5 (M3) and 6 (M4). The mixture was magnetically stirred for $10 \mathrm{~min}$ at room temperature, then heated up to $200{ }^{\circ} \mathrm{C}$ at a rate of $4{ }^{\circ} \mathrm{C} / \mathrm{min}$ and was kept at this temperature for $40 \mathrm{~min}$. Afterwards, the reaction was heated up to $310^{\circ} \mathrm{C}$ at a rate of $3{ }^{\circ} \mathrm{C} / \mathrm{min}$ and kept at that temperature for 60 min (M1-M4), $30 \mathrm{~min}$ (M2.1) or $90 \mathrm{~min}$ (M2.2). After cooling, the IO-OA NPs were magnetically washed five times with $2.5 \mathrm{~mL}$ of 1:4 hexane:acetone, $3.75 \mathrm{~mL}$ of isopropanol and $5 \mathrm{~mL}$ of ethanol. Particles were centrifuged at 1,400 rpm for $2 \mathrm{~min}$, the supernatant was decanted and particles were resuspended in hexane. Finally, they were precipitated again with ethanol and finally dissolved and preserved in $8 \mathrm{~mL}$ of hexane.

\subsection{Silanization of IO NPs by Ligand Exchange of Oleic Acid to APS}

Oleic acid on the surface of the IO NPs was exchanged for silane by the fractionated drop method using APS. Specifically, $1 \mathrm{~mL}$ of IO NPs (sample M2.1) was dissolved in $21.2 \mathrm{~mL}$ of toluene, followed by the addition of $3.5 \mu \mathrm{L}$ of acetic acid, the stirring and the heating of the solution to $70{ }^{\circ} \mathrm{C}$. In another flask, containing $20 \mathrm{~mL}$ of toluene, different concentrations of APS were added: $0.14 \mathrm{mmol}$ (S1), $1.38 \mathrm{mmol}$ (S2), $5.73 \mathrm{mmol}$ (S3). The APS/toluene solution was then added to the IO NPs solution at a drop rate of $0.16 \mathrm{~mL} / \mathrm{min}$. After the dropping, the reaction was kept at $70{ }^{\circ} \mathrm{C}$ for $24 \mathrm{~h}$ (for all samples) and $48 \mathrm{~h}$ (S2.1). After cooling at room temperature, the silane-IO NPs were magnetically washed several times with ethanol and isopropanol and then were resuspended in $35 \mathrm{~mL}$ of toluene (Fig. 1A).

\subsection{Pegylation of Silane-IO NPs}

PEG-dicarboxylic acid was incorporated to the silane-IO NPs by an amide linkage between the terminal primary amine on the silane and the terminal carboxylic acid on PEG-diCOOH. Specifically, $35 \mathrm{~mL}$ silane-IO NPs (sample S2) and $0.544 \mathrm{~g}$ of PEG-dicarboxylic acid were mixed at $70{ }^{\circ} \mathrm{C}$, then it was heated to reflux for $2 \mathrm{~h}$ under nitrogen atmosphere (Fig. 1B). The toluene of $7 \mathrm{~mL}$ of the PEG-silane IO NPs was eliminated with a rotary evaporator and then the particles were resuspended in a $0.07 \mathrm{M} \mathrm{NaCl}$ solution (sample S2.1).

\subsection{Amination of PEG-Silane-IO NPS}

Totally $28 \mathrm{~mL}$ of the PEG-silane-IO NPs was mixed with $15.3 \mu \mathrm{L}$ of EDEA at $70{ }^{\circ} \mathrm{C}$, then the solution was heated to reflux for $2 \mathrm{~h}$ under nitrogen atmosphere. After cooling at room temperature, the NPs were magnetically washed several times with ethanol and isopropanol and then toluene was evaporated on a rotary evaporator. Subsequently, half were re-suspended in distilled water and half in toluene and labeled as W1 and T1, respectively (Fig. 1C).

\subsection{Characterization techniques}

\subsubsection{FT-IR Spectroscopy}

Infrared spectroscopy, in a PERKIN-ELMER Spectrum 100 spectrometer, was used to study changes in functional groups regarding the synthesis of PEG-dicarboxylic acid, IO-OA NPs synthesis and each surface modification of the IO NPs. Samples were compressed to a pellet with $\mathrm{KBr}$ and scanned from 4,000 to $400 \mathrm{~cm}^{-1}$ using pure $\mathrm{KBr}$ as blank.

2.7.2 STEM (Scanning Transmission Electron Microscopy)

Particles size and morphology were studied by 


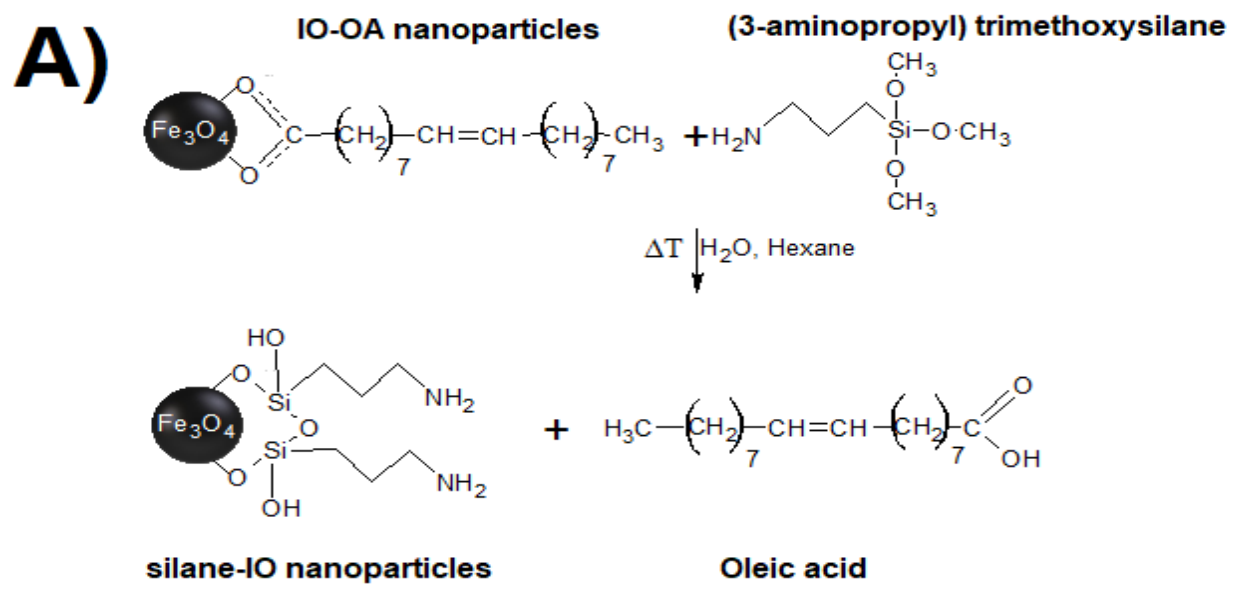

B)
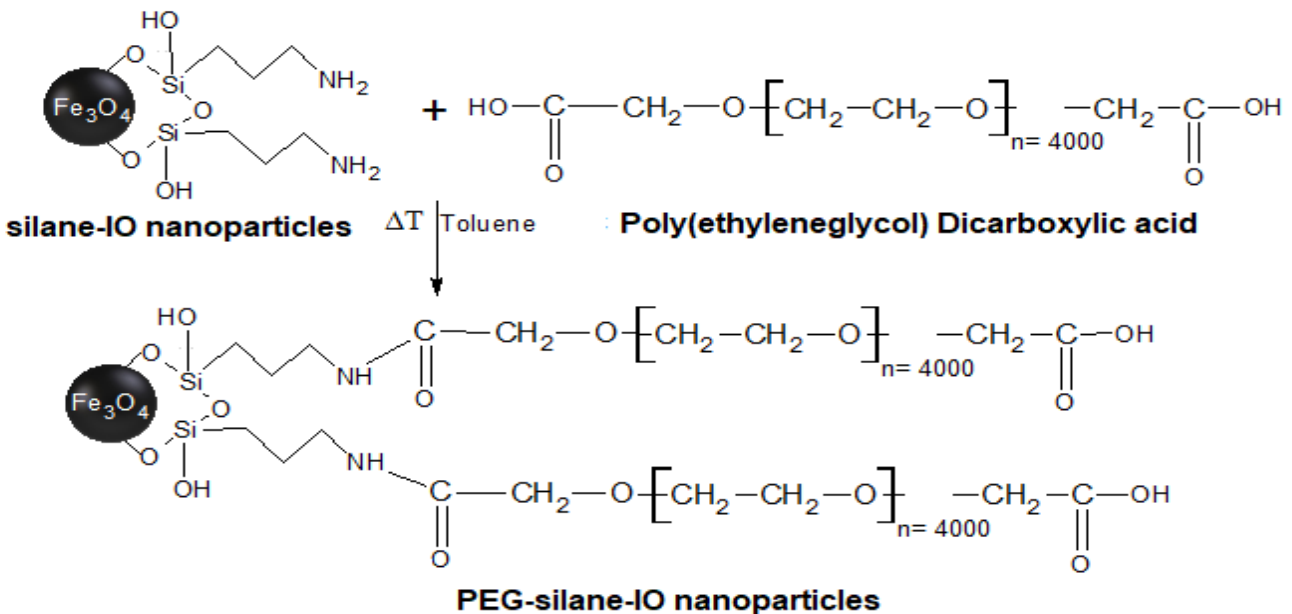

C)

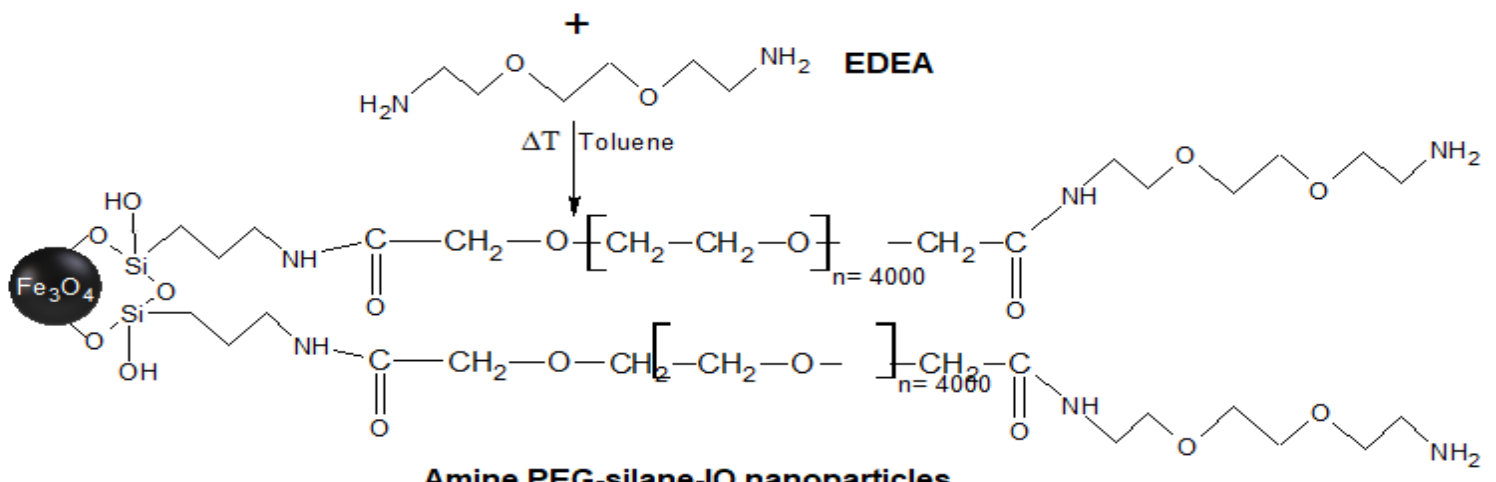

Amine PEG-silane-IO nanoparticles

Fig. 1 Schematic representation of the synthesis of IO NPs coated with amine terminated PEG-silane. Ligand exchange reaction of oleic acid to APS on the IO NPs (A), pegylation of silane-IO NPs (B) and incorporation of amine groups to PEG-silane-IO NPs with EDEA (C), both by amide reactions. 
STEM using a CARL ZEISS EVO HD-15 microscope and a Jeol JSM-7800 F microscope (only for IO-OA NPs). Samples were prepared by depositing a drop (5 $\mu \mathrm{L}$ ) on 400 mesh Formvar carbon coated copper TEM grid, except for IO-OA NPs which were deposited on a 400 mesh Lacey carbon coated copper TEM grid. Mean particle size and size distribution were evaluated by measuring the largest internal dimension of at least 200 particles using the ImageJ software. Particle size distribution normality was corroborated with an Anderson-Darling test $(p>0.05)$.

\subsubsection{Dynamic Light Scattering (DLS)}

The hydrodynamic size of the particles was acquired with a Malvern Zetasizer Nano. Samples were filtered using a $0.2 \mu \mathrm{m}$ nylon filter. IO-OA and silane-IO were suspended in toluene; silane-IO were measured after 1, 3.5, 6, 12, 24 and 49 hours of reaction time. Meanwhile, for amine PEG-silane-IO NPs were first dissolved in toluene and water $(\mathrm{pH}=9)$ and then the colloidal stability of was studied in deionized water, $0.07 \mathrm{M} \mathrm{NaCl}$ and $1 \mathrm{x}$ PBS, as a function of time from 0 to 30 days. Data were obtained from the size distribution by number of the instrument.

\section{Experimental Results}

\subsection{FT-IR Analysis}

PEG was successfully oxidized to PEG-diacid as confirmed from the appearance of a band at 1,747 $\mathrm{cm}^{-1}$, characteristic of the $-\mathrm{C}=\mathrm{O}$ vibration of $-\mathrm{COOH}$ in the FT-IR spectrum (Fig. 2). A 90 to $99 \%$ conversion of hydroxyl groups to carboxylic acids was obtained when compared to $1.0 \mathrm{mmol} \mathrm{COOH} / \mathrm{g}$ PEG-diacid solution considering all hydroxyl groups available were converted and a mean molecular weight of 4,000.

Fig. 3A shows the FT-IR spectra of IO-OA (black) and silane-IO NPs (red). IO-OA NPs relevant vibrational bands are at $1,640 \mathrm{~cm}^{-1}$, corresponding to the

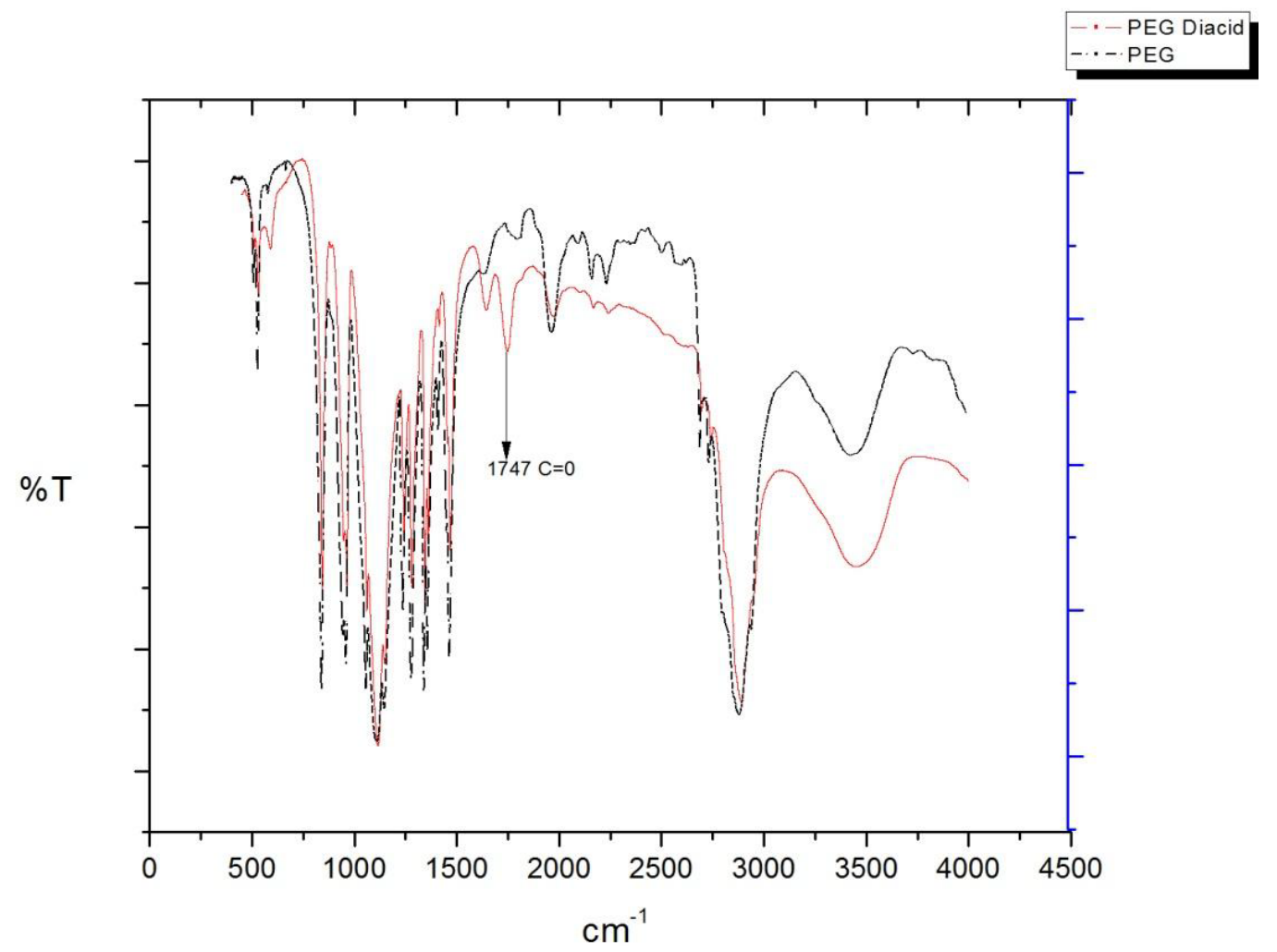

Fig. 2 Oxidation of hydroxyl groups in PEG (black line) to carboxylic acids to form PEG-diacid (red line). 
chelating bidentate bond between oleic acid and Fe atoms, at 2,924 $\mathrm{cm}^{-1}$ and at $2,854 \mathrm{~cm}^{-1}$, corresponding to asymmetric and symmetric $\mathrm{CH}_{2}$ vibration [12, 17 , 18]. The ligand exchange reaction of oleic acid molecules for APS molecules is confirmed mainly by bands at $1,124 \mathrm{~cm}^{-1}$ and $500 \mathrm{~cm}^{-1}$, characteristic of Si-O-Si asymmetric stretching vibration and of Fe-O-Si, respectively. Furthermore, peaks at $1,637 \mathrm{~cm}^{-1}, 1,260$ $\mathrm{cm}^{-1}$ and $2,931 \mathrm{~cm}^{-1}$ correspond to $\mathrm{N}-\mathrm{H}$ bending vibration, $\mathrm{C}-\mathrm{N}$ bond and asymmetric $\mathrm{CH}_{2}$ bond $[3,19]$.

On the other hand, the FT-IR spectra of PEG-silane-IO (black) and amine PEG-silane-IO (red) NPs are shown in Fig. 3B. Both spectra show broad peaks from 2,845 $\mathrm{cm}^{-1}$ to 2,945 $\mathrm{cm}^{-1}$ probably attributed to the overlapping $\mathrm{CH}_{2}$ peaks from the silane (peaks at 2,925 $\mathrm{cm}^{-1}$ and 2,852 $\mathrm{cm}^{-1}$ ) and the PEG-diacid (peak at 2,894 $\mathrm{cm}^{-1}$ ). Additionally, the $1,124 \mathrm{~cm}^{-1} \mathrm{Si}-\mathrm{O}-\mathrm{Si}$ peak from the silane-IO NPs was shifted to $1,109 \mathrm{~cm}^{-1}$ and also broadened probable due to the C-O-C bond on PEG-diacid [15]. The Fe-O-Si peak at $500 \mathrm{~cm}^{-1}$ shifted to $620 \mathrm{~cm}^{-1}$ in both spectra [20]. The utmost differences between these two spectra are: (a) the disappearance of peak at 1,748 $\mathrm{cm}^{-1}$ that corresponds to the carbonyl bond from the PEG-diacid's available carboxylic acid group due to the formation of a new amide linkage; and (b) the appearance of a band at 1,641 $\mathrm{cm}^{-1}$, characteristic of the $\mathrm{C}(=\mathrm{O})-\mathrm{NH}$ vibration in PEG-silane-IO NPs, which shifts to $1,620 \mathrm{~cm}^{-1}$ in amino PEG-silane-IO NPs as a consequence of the recently formed amide bond and the additional $\mathrm{NH}_{2}$. Furthermore, peaks at, 1342, 1279, 1241, 961, 842, 529 and $509 \mathrm{~cm}^{-1}$, indicate that the PEG grafted onto the particle surface is crystalline, as described previously [13]. Finally, the spectra of PEG-silane-IO NPs in toluene (sample S2) and 0.07 $\mathrm{M} \mathrm{NaCl}$ (sample S2.1) are very similar (data not shown).

\subsection{Effect of Oleic Acid/Iron Oleate Molar Ratio on IO-OA Nanoparticles' Formation}

In the thermal decomposition method, the molar ratio oleic acid to iron oleate and correct timing of the nucleation period $\left(200{ }^{\circ} \mathrm{C}\right)$ and the growth stage $\left(310^{\circ} \mathrm{C}\right)$, allow precise control in particles size and dispersity [12, 17]. Fig. 4 shows that, when maintaining the growth stage for $60 \mathrm{~min}$, the ideal oleic acid/iron oleate molar ratio is 3.0 because it produces IO-OA NPs of $10.93 \pm 1.31 \mathrm{~nm}$; meanwhile, lowering the molar ratio to 1.5 produces $12.43 \pm 2.05$ $\mathrm{nm}$ NPs since not enough oleic acid is available to fully coat the particles which counters the magneto dipole-dipole interactions within particles. Contrarily, excessive oleic acid, when using 4.5 and 6.0 molar ratios, increases both the particles size by enlarging the oleic acid shell and their dispersity due to the overcovering the growth sites of some particles, producing sizes of $14.28 \pm 1.68 \mathrm{~nm}$ and $16.68 \pm 4.43$ $\mathrm{nm}$, respectively.

Next, we fixed the oleic acid/iron oleate molar ratio to 3.0 and tested with $60 \pm 30 \mathrm{~min}$ at the growth stage. Setting the growth time to $30 \mathrm{~min}$ formed $10.11 \pm$ $1.020 \mathrm{~nm}$ NPs (sample M2.1) and setting to $90 \mathrm{~min}$ produced particles of $15.05 \pm 0.88 \mathrm{~nm}$ (sample M2.2). This indicates that increased growth times lead to lower standard deviations but to higher mean diameters (Figs. 4D and 4F), which agrees with previous reports $[10,12]$. Nanoparticles are considered monodisperse if $\log \sigma<0.2$ which was true for all the synthesized nanoparticles with 3.0 as oleic acid/iron oleate molar ratio. Particles with the lowest mean diameter and $\log \sigma$ were chosen for silanization (sample M2.1).

\subsection{APS Concentration and Reaction Time Effect on IO Nanoparticles'Silanization}

Particle silanization is particularly difficult due to the formation of thick silane layers on the surface and/or the formation of free silica particles. To control these phenomena, we used fractionated dropping of APS because it can ensure that the concentration of APS monomers, as a function of time, stays above the heterogeneous nucleation concentration and below the 

Resection Grade Improvement
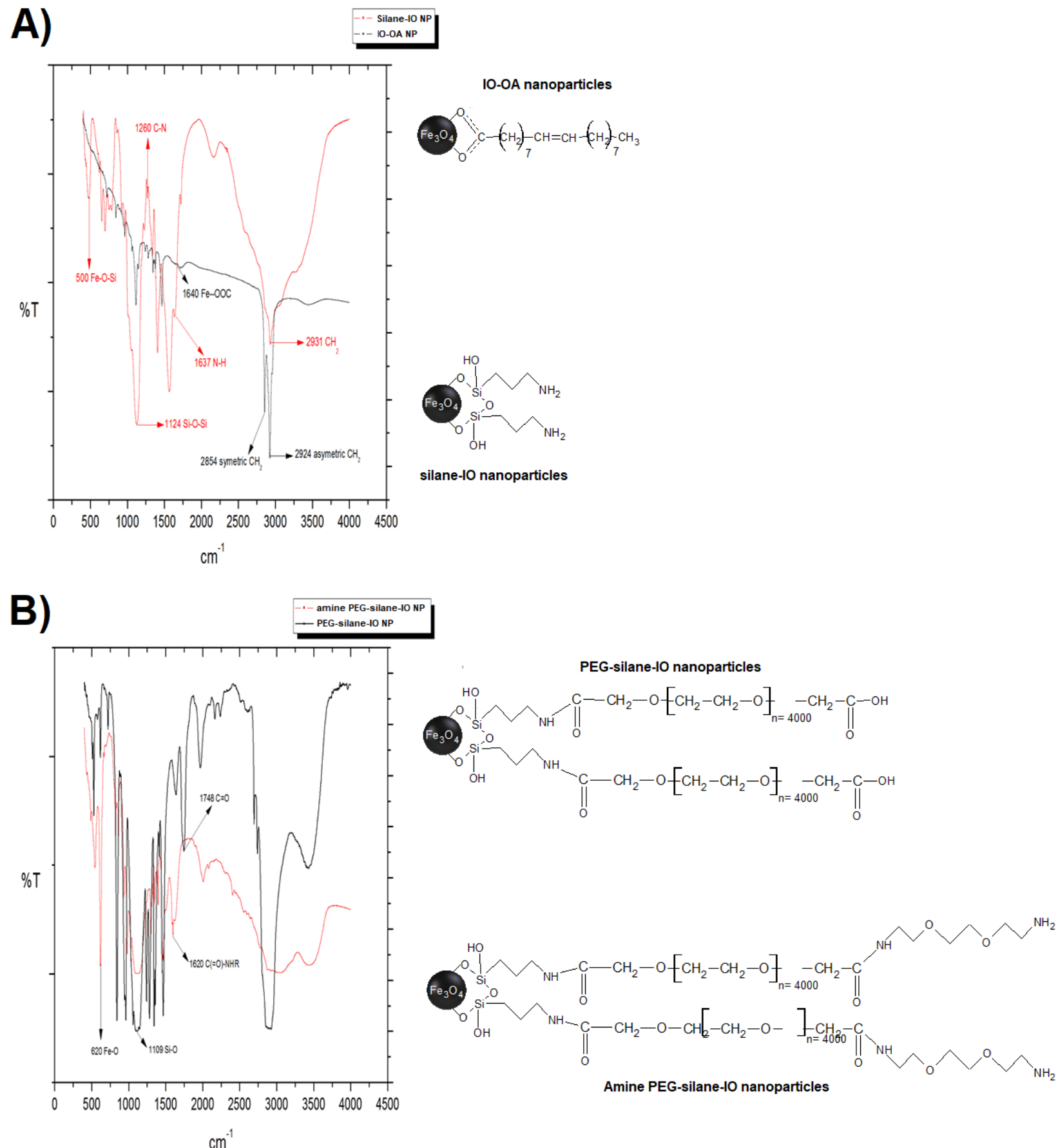

Fig. 3 FT-IR spectra of IO NPs with different coatings. IO-OA (A black line), silane-IO (A red line), PEG-silane-IO (B black line) and amine PEG-silane-IO (B red line). 


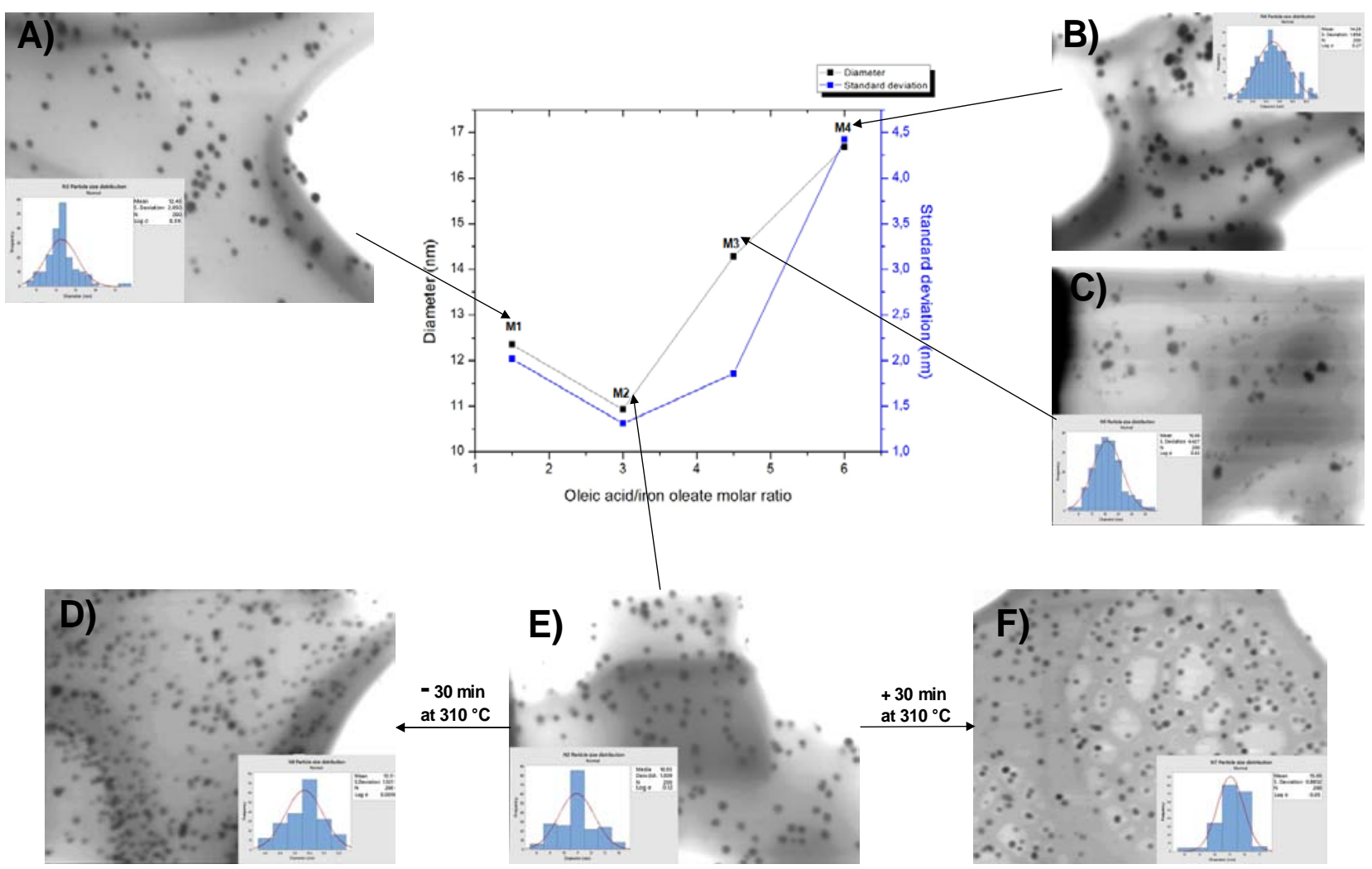

Fig. 4 STEM micrographs of IO-OA NPs generated with different oleic acid/iron oleate molar ratios: 1.5 (A; sample M1), 6.0 (B, sample M4), 4.5 (C; sample M3) and 3.0 (D-F; samples M2, M2.1, M2.2). Reflux reaction time at $310{ }^{\circ} \mathrm{C}$ was 60 min except for $D(30 \mathrm{~min})$ and $\mathrm{F}$ (90 min). Histograms show each NPs' size normal distribution where: $\mathrm{N}=$ number of particles measured; $\log \sigma=$ standard deviation logarithm.

homogenous nucleation concentration [21]. DLS measurements, in Fig. 5A (top), evidences a similar effect as when coating with oleic acid, where lacking (0.14 mmol) or excess $(5.73 \mathrm{mmol})$ of APS results in higher nanoparticles' diameter (37.4 nm and $87.2 \mathrm{~nm}$, respectively) compared to the $19.7 \mathrm{~nm}$ obtained with $1.38 \mathrm{mmol}$ of APS (sample S2) and fixed reaction time of $24 \mathrm{~h}$.

Subsequently, we fixed the APS to $1.38 \mathrm{mmol}$ and proceeded to evaluate the silane coating on the NPs at different reaction times (Fig. 5, bottom A), obtaining increasing silane diameters up to $6 \mathrm{~h}$ of reaction (243.1 nm) which then decreases and stabilizes until $24 \mathrm{~h}(19.7 \mathrm{~nm})$ and finally slightly increases at $48 \mathrm{~h}$ $(39.9 \mathrm{~nm})$, which agrees with a previous report [22]. The STEM micrograph of sample S2 $(1.38 \mathrm{mmol}$ of APS, 24 h reaction time) shows that the silane-IO NPs have a diameter of $15.22 \pm 3.7 \mathrm{~nm}$ (Fig. $5 \mathrm{~B}$ ), which is larger than when coated with oleic acid probably as a result of the formation of multilayers [3, 22]. Sample S2 was chosen for pegylation and amination.

\subsection{Colloidal Stability of Amine PEG-Silane-IO NPs}

Fig. 6A shows that the PEG-silane-IO NPs diameter as a function of time, dissolved in $1 \mathrm{X}$ PBS, $0.07 \mathrm{M}$ $\mathrm{NaCl}$ and deionized water, follows a sigmoid curve behavior with notorious increments within the first 14 days and then reaching stability up to day 30. Albeit, the particles diameter increases $7.7 \%, 13 \%$ and $9.7 \%$, (from day 0 to day 30) when dissolved in 1X PBS, $0.07 \mathrm{M} \mathrm{NaCl}$ and deionized water, no significant agglomeration was observed within time nor the solutions used.

In Fig. 6B we observe that the NPs diameter decreases to $12.09 \pm 2.3 \mathrm{~nm}$ as compared to when only silanized (15.22 $\pm 3.7 \mathrm{~nm}$ ), hence meaning pegylation 
A)

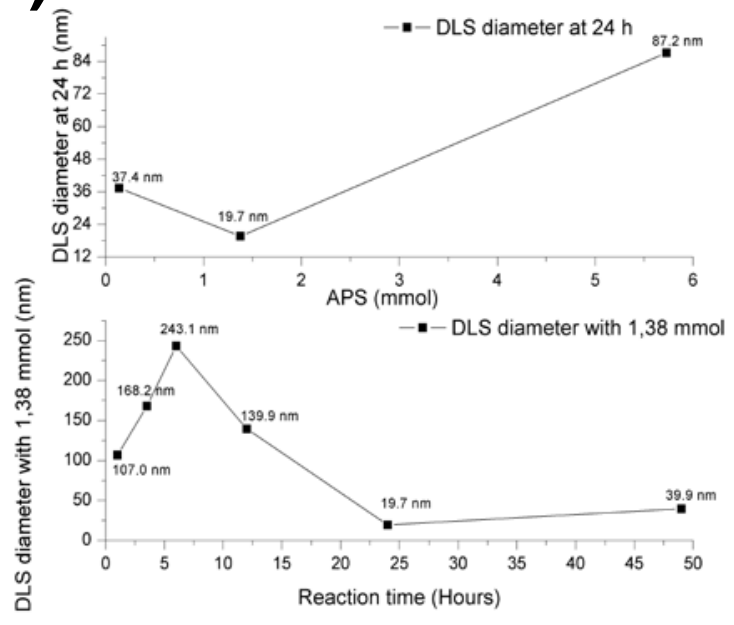

\section{B)}

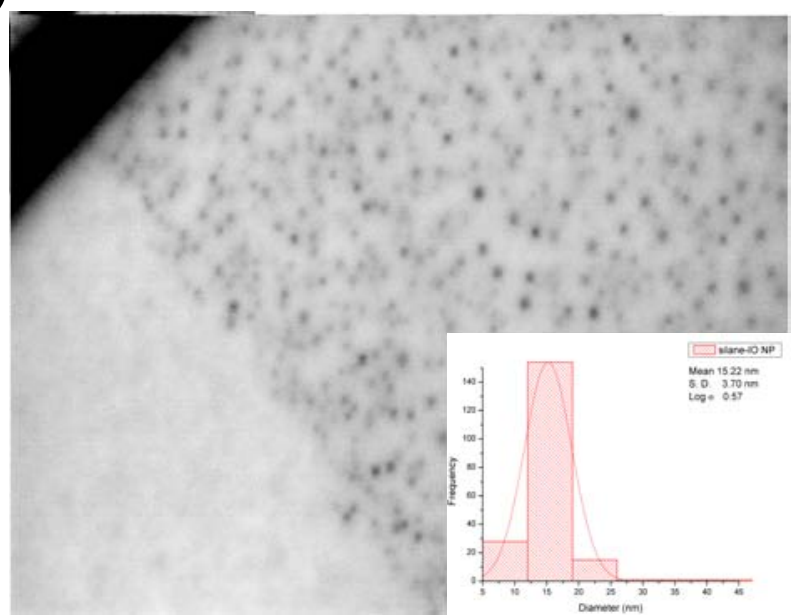

Fig. 5 silane-IO nanoparticles DLS diameter as a function of APS concentration (top A) and reaction time (bottom A). STEM micrograph of silane-IO NP sample S2 with $1.38 \mathrm{mmol}$ of APS after 24 hours of reaction (B). Histogram shows nanoparticles' size normal distribution where: $\mathrm{N}=$ number of particles measured; Log $\sigma=$ standard deviation logarithm.

A)

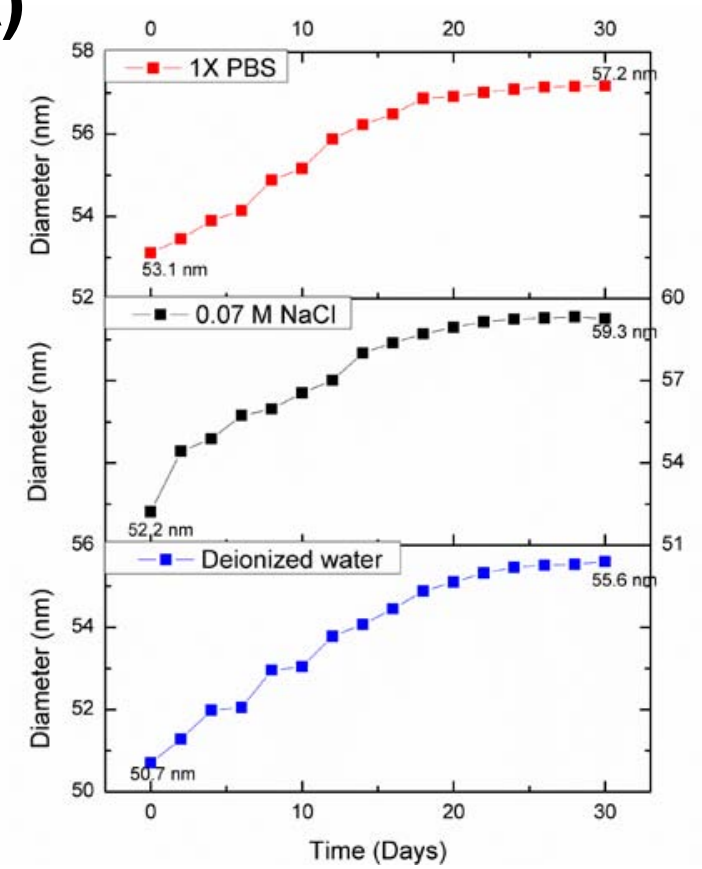

B)

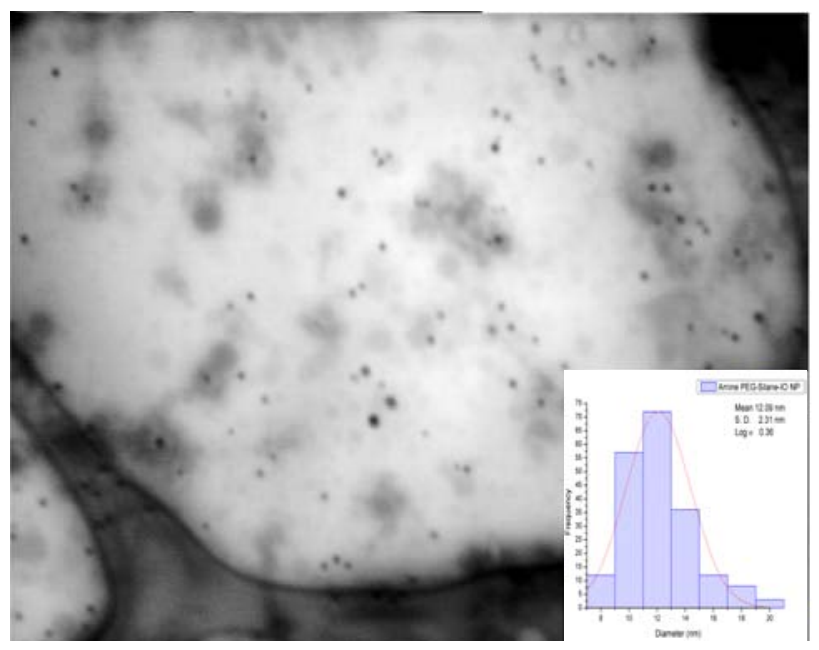

Fig. 6 Colloidal stability of amine-PEG-silane-IO NP, as a function of time, dissolved in 1xPBS (top A), 0.07 M NaCl (middle A) and deionized water (bottom A). STEM micrograph of amine PEG-silane-IO NP sample W1 (B). Histogram shows nanoparticles' size normal distribution where: $N=$ number of particles measured; Log $\sigma=$ standard deviation logarithm. 

Resection Grade Improvement

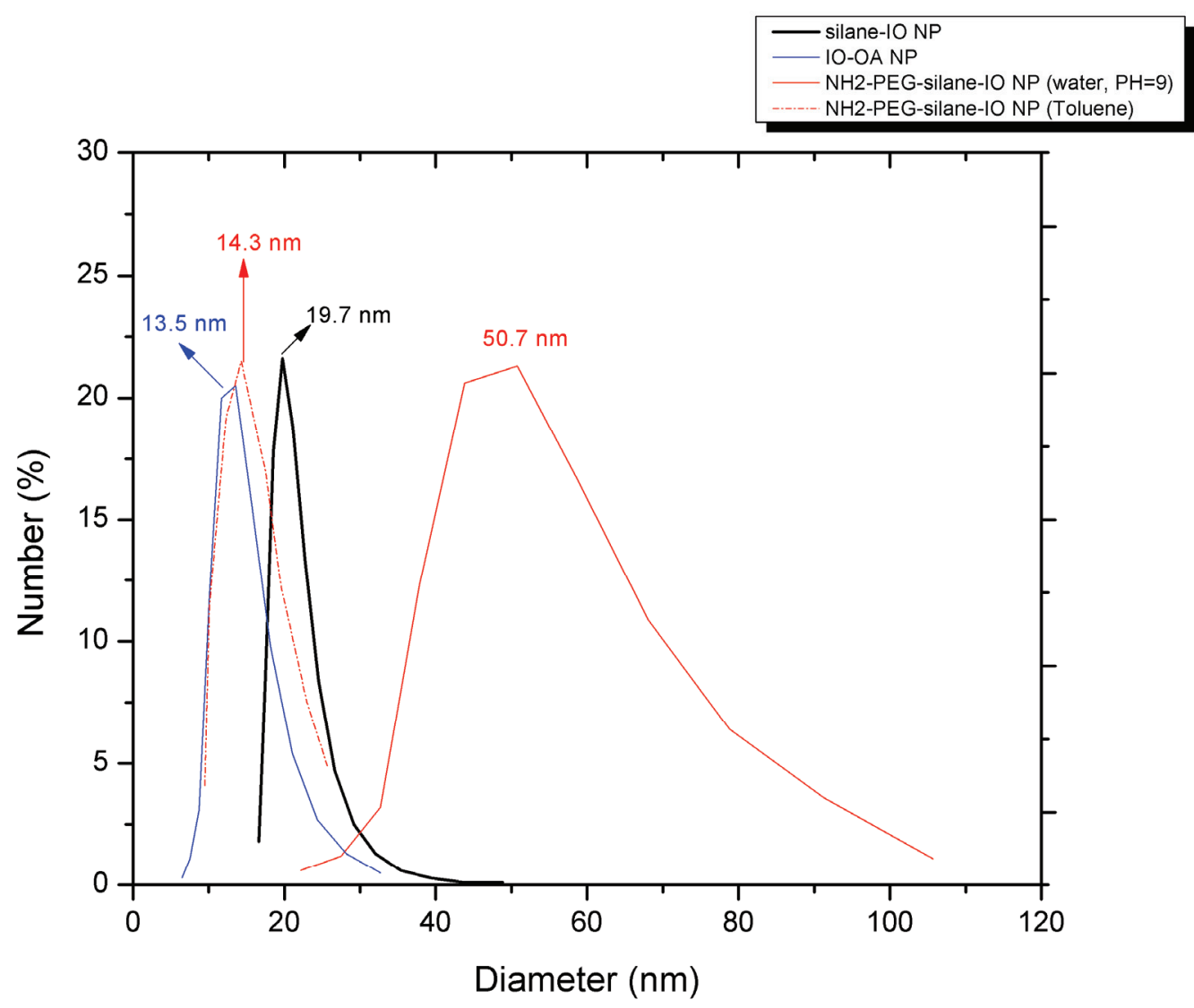

Fig. 7 Hydrodynamic size measured by DLS of IO NPs with different surface coatings. 


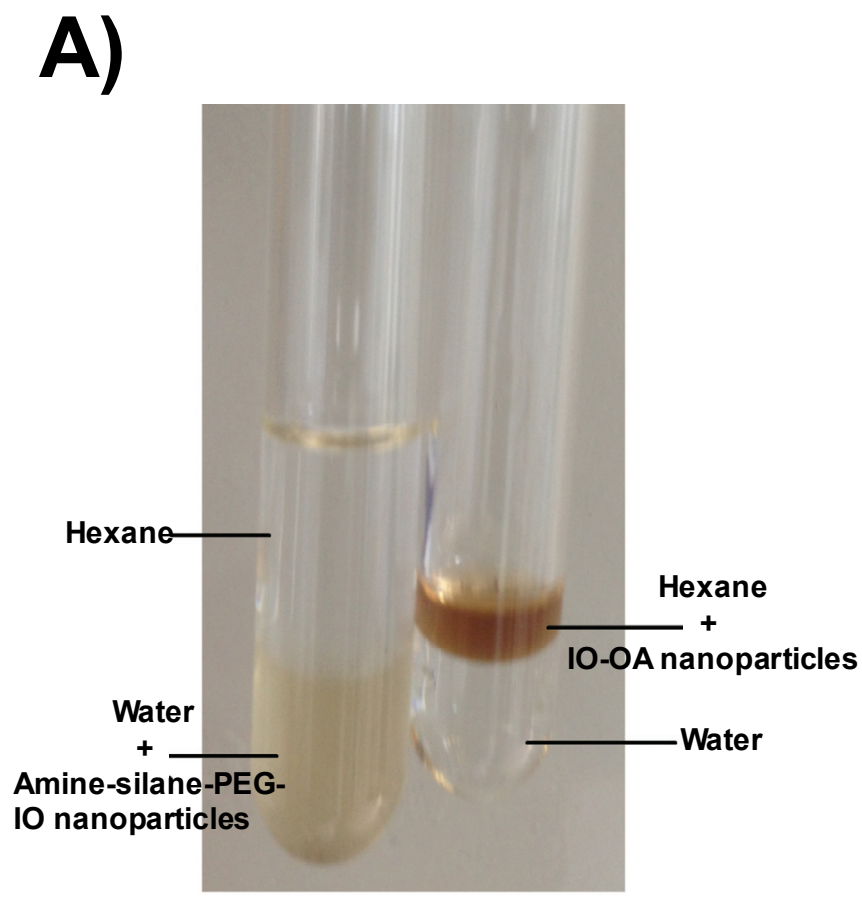

B)

Fig. 8 IO nanoparticles solubility: amine silane pegylated dissolved in water (left A) and oleic acid coated dissolved in hexane (right A). Magnetic response of Amine PEG-IO NP dissolved in water (B).

grants better stability to the IO NPs and a possible decoupling of some silane layers on the particles surface during pegylation [13]. Furthermore, water molecules trapped within the PEG coating on the particles increase its hydrodynamic size to $58.28 \mathrm{~nm}$ since measuring the amine PEG-silane IO NP in toluene gives a diameter of only $14.3 \mathrm{~nm}$ (Fig. 7).

Pegylation not only allowed the IO nanoparticles solubility in water, which was not possible when coated with oleic acid (Fig. 8A), but also did not impact their magnetic response since the particles could be quickly attached by an external magnet and dispersed again after removing it (Fig. 8B).

\section{Conclusions}

In conclusion, the synthesis of monodisperse iron oxide NPs using the thermal decomposition method, and their silane ligand exchange reaction through the fractionated drop method, are affected both by the stabilizer concentration with respect to the particles' concentration and singularly by the reaction time during the growth stage or the nucleation stage, respectively. After pegylation and amination, the iron oxide NPs exhibited colloidal stability in deionized water, $0.07 \mathrm{M} \mathrm{NaCl}, 1 \mathrm{X}$ PBS and good dispersibility in organic solvents like toluene. Despite the different molecules attached, the nanoparticles possess a thin shell, desired size range to penetrate the blood-brain barrier, excellent magnetic properties, and proper amine terminated groups to permit the covalent attachment of other molecules to their surface, opening doors to numerous bio-applications. Specifically to our interests, the synthesized nanoparticles could allow us astrocytoma resection grade improvement, including GBM, after the incorporation of Chlorotoxin and a fluorescent 
molecule to their surface.

\section{Acknowledgments}

This work was funded by the Universidad Iberoamericana León. The authors wish to express their appreciation to Universidad de Guanajuato for STEM, DLS and FT-IR measurements, to Hospital Regional de Alta Especialidad del Bajío for allowing the attendance to brain surgeries, to Ph.D. Ibarra-Sánchez and Ph.D. Juan Vargas Mancilla for their suggestions and guidance.

\section{References}

[1] Giese, A., Bjerkvig, R., Berens, M. E., and Westphal, M. 2003. "Cost of Migration: Invasion of Malignant Gliomas and Implications for Treatment.” J Clin Oncol. 21: 1624-36.

[2] Lara-Velazquez, M., Al-Kharboosh, R., Jeanneret, S., Vazquez-Ramos, C., Mahato, D., Tavanaiepour, D., Rahmathulla, G., and Quinones-Hinojosa, A. 2017. "Advances in Brain Tumor Surgery for Glioblastoma in Adults.” Brain Sciences 7: 166.

[3] Liu, F., Niu, F., Peng, N., Su, Y., and Yang, Y. 2015. "Synthesis, Characterization, and Application of $\mathrm{Fe}_{3} \mathrm{O}_{4} @ \mathrm{SiO}_{2}-\mathrm{NH}_{2}$ Nanoparticles.” RSC Advances 5: 18128-36.

[4] Grande, A. 2007. "Nanotecnología Y Nanopartículas Magnéticas: la Física Actual en Lucha Contra la Enfermedad.” Rev. R. Acad. Cienc. Exact. Fís. Nat. 101: 321-7.

[5] Veiseh, O., Sun, C., Gunn, J., Kohler, N., Gabikian, P., Lee, D., Bhattarai, N., Ellenbogen, R., Sze, R., Hallahan, A., Olson, J., and Zhang, M. 2005. "Optical and MRI Multifunctional Nanoprobe for Targeting Gliomas.” Nano Lett. 5: 1003-8.

[6] Sun, C., Du, K., Fang, C., Bhattarai, N., Veiseh, O., Kievit, F., Stephen, Z., Lee, D., Ellenbogen, R. G., Ratner, B., and Zhang, M. 2010. "PEG-Mediated Synthesis of Highly Dispersive Multifunctional Superparamagnetic Nanoparticles: Their Physicochemical Properties and Function in Vivo.” ACS Nano. 4: 2402-10.

[7] Veiseh, O., Sun, C., Fang, C., Bhattarai, N., Gunn, J., Kievit, F., Du, K., Pullar, B., Lee, D., Ellenbogen, R. G., Olson, J., and Zhang, M. 2009. "Specific Targeting of Brain Tumors with an Optical/Magnetic Resonance Imaging Nanoprobe across the Blood-Brain Barrier.” Cancer Res. 69: 6200-7.

[8] Bhattarai, N., Matsen, F. A., and Zhang, M. 2005. "PEG-Grafted Chitosan as an Injectable Thermoreversible
Hydrogel.” Macromolecular Bioscience 5: 107-11.

[9] Chen, Y.-C., Chang, W.-H., Wang, S.-J., and Hsieh, W.-Y. 2012. "Fluorescent Magnetic Nanoparticles with Specific Targeting Functions for Combinded Targeting, Optical Imaging and Magnetic Resonance Imaging.” Journal of Biomaterials Science, Polymer Edition 23: 1903-22.

[10] Chen, Z. 2012. "Size and Shape Controllable Synthesis of Monodisperse Iron Oxide Nanoparticles by Thermal Decomposition of Iron Oleate Complex.” Synthesis and Reactivity in Inorganic, Metal-Organic, and Nano-Metal Chemistry 42: 1040-6.

[11] Ibarra-Sánchez, J. J., Fuentes-Ramírez, R., Roca, A. G., del Puerto Morales, M., and Cabrera-Lara, L. I. 2013. "Key Parameters for Scaling up the Synthesis of Magnetite Nanoparticles in Organic Media: Stirring Rate and Growth Kinetic." Industrial \& Engineering Chemistry Research 52: 17841-7.

[12] Kwon, S. G., Piao, Y., Park, J., Angappane, S., Jo, Y., Hwang, N. M., Park, J. G., and Hyeon, T. 2007. "Kinetics of Monodisperse Iron Oxide Nanocrystal Formation by ‘Heating-Up’ Process.” J Am Chem Soc. 129: 12571-84.

[13] Barrera, C., Herrera, A. P., and Rinaldi, C. 2009. "Colloidal Dispersions of Monodisperse Magnetite Nanoparticles Modified with Poly(Ethylene Glycol).” J Colloid Interface Sci. 329: 107-13.

[14] Karakoti, A. S., Das, S., Thevuthasan, S., and Seal, S. 2011. "PEGylated Inorganic Nanoparticles.” Angewandte Chemie International Edition 50: 1980-94.

[15] Barrera, C., Herrera, A. P., Bezares, N., Fachini, E., Olayo-Valles, R., Hinestroza, J. P., and Rinaldi, C. 2012. "Effect of Poly(Ethylene Oxide)-Silane Graft Molecular Weight on the Colloidal Properties of Iron Oxide Nanoparticles for Biomedical Applications.” J Colloid Interface Sci. 377: 40-50.

[16] Lele, B. S., and Kulkarni, M. G. 1998. "Single Step Room Temperature Oxidation of Poly(Ethylene Glycol) to Poly(Oxyethylene)-Dicarboxylic Acid.” Journal of Applied Polymer Science 70: 883-90.

[17] Zhang, L., He, R., and Gu, H.-C. 2006. "Oleic Acid Coating on the Monodisperse Magnetite Nanoparticles.” Applied Surface Science 253: 2611-7.

[18] Ozel, F., Kockar, H., Beyaz, S., Karaagac, O., and Tanrisever, T. 2013. "Superparamagnetic Iron Oxide Nanoparticles: Effect of Iron Oleate Precursors Obtained with a Simple Way." Journal of Materials Science: Materials in Electronics 24: 3073-80.

[19] Palimi, M. J., Rostami, M., Mahdavian, M., and Ramezanzadeh, B. 2014. "Surface Modification of $\mathrm{Fe}_{2} \mathrm{O}_{3}$ Nanoparticles with 3-Aminopropyltrimethoxysilane (APTMS): An Attempt to Investigate Surface Treatment on Surface Chemistry and Mechanical Properties of 
Resection Grade Improvement

Polyurethane/ $\mathrm{Fe}_{2} \mathrm{O}_{3}$ Nanocomposites.” Applied Surface Science 320: 60-72.

[20] Bloemen, M., Brullot, W., Luong, T. T., Geukens, N., Gils, A., and Verbiest, T. 2012. Journal of Nanoparticle Research 14: 1100.

[21] Ding, H. L., Zhang, Y. X., Wang, S., Xu, J. M., Xu, S. C., and Li, G. H. 2012. " $\mathrm{Fe}_{3} \mathrm{O}_{4} @ \mathrm{SiO}_{2}$ Core/Shell
Nanoparticles: The Silica Coating Regulations with a Single Core for Different Core Sizes and Shell Thicknesses.” Chemistry of Materials 24: 4572-80.

[22] Liu, Y., Li, Y., Li, X.-M., and He, T. 2013. "Kinetics of (3-Aminopropyl)triethoxylsilane (APTES) Silanization of Superparamagnetic Iron Oxide Nanoparticles.” Langmuir 29: $15275-82$. 